\title{
The effect of systemic cocaine on spontaneous and nociceptively evoked activity of neurons in the medial and lateral thalamus
}

\author{
Carl R. Belczynski Jr. ${ }^{3}$, Antti Pertovaara ${ }^{4,}$, Thomas J. Morrow ${ }^{2,4}$ and Kenneth L. Casey ${ }^{1,2,4}$ \\ Departments of ${ }^{1}$ Neurology, ${ }^{2}$ Physiology and ${ }^{3}$ Neuroscience Program, University of Michigan, and ${ }^{4}$ Neurology Research Laboratories, VA \\ Medical Center, Ann Arbor, MI 48105 (U.S.A.)
}

(Accepted 30 January 1990)

Key words: Cocaine; Analgesia; Unit analysis; Electrophysiology; Thalamus; Nociception; Somatosensory

\begin{abstract}
In rats, analgesic doses of cocaine $(25 \mathrm{mg} / \mathrm{kg}$, i.p.) suppressed the responses of 20 of 22 medial and lateral thalamic neurons to reticular formation and somatic noxious stimuli. The responses of 7 of 8 lateral thalamic neurons to innocuous somatic stimuli were unaffected or enhanced. The suppression of thalamic responses to noxious stimuli may be part of the mechanism underlying cocaine analgesia.
\end{abstract}

Recently, this laboratory demonstrated that systemically administered cocaine is a rapidly acting $(<5 \mathrm{~min})$, non-sedative, central analgesic in the rat ${ }^{11}$. Cocaine analgesia is not reversed by naloxone, is blocked by dopamine antagonists, and appears to be independent of cardiovascular or local anaesthetic actions of the compound ${ }^{11}$.

Centrally acting analgesics may enhance bulbospinal inhibition of spinothalamic tract excitation ${ }^{1,7,8}$. The spinothalamic tract projects to the medial thalamus ${ }^{10,18}$, and a significant proportion of cells in the medullary reticular formation (MRF) and medial thalamus respond exclusively or differentially to noxious or escape-eliciting stimuli $^{3-6,9,14,17}$. We have recently demonstrated that analgesic doses of cocaine increase the spontaneous discharge and decrease the nociceptively evoked activity of (MRF) neurons with axonal projections to the medial thalamus ${ }^{2}$. We conducted this preliminary study to determine whether an analgesic dose of cocaine produces an opiate-independent suppression of medial thalamic neuronal responses to somatic noxious and MRF electrical stimuli, and to compare this effect with that observed for similar responses of ventrobasal thalamic neurons.

Twenty-two adult, male Sprague-Dawley rats (250$400 \mathrm{~g})$ were anaesthetized with chloral hydrate (400 $\mathrm{mg} / \mathrm{kg}$, i.p.) with supplemental doses sufficient to suppress flexion reflexes. End-tidal $\mathrm{CO}_{2}$ was maintained between 3.5 and $4.5 \%$ and the rectal temperature between 36.5 and $38.5^{\circ} \mathrm{C}$. Animals were placed in a spinal stereotaxic frame according to the atlas of Paxinos and Watson ${ }^{15}$. Insulated stainless-steel microelectrodes
(8-22 $\mathrm{M} \Omega$ at $100 \mathrm{~Hz})$ were directed toward either the lateral ventrobasal thalamus (VB; A $5.2 \mathrm{~mm}, \mathrm{~L} \pm 2.8$ $\mathrm{mm}$, D $5.0 \mathrm{~mm}$ ) or the medial thalamic centromedianparafascicularis complex (CM-PF; A $4.8 \mathrm{~mm}, \mathrm{~L}+1.5$ $\mathrm{mm}, \mathrm{D} 4.0 \mathrm{~mm}$ ). Action potentials from single neurons triggered pulses from a window discriminator; these pulses, along with digitized outputs from all stimulating devices, were fed to a minicomputer system. Spike isolation and amplitude were continuously monitored throughout each experiment and were typically maintained for 30-90 min. Transcutaneous innocuous and noxious electrical $(5-10 \mathrm{~mA}, 5 \mathrm{~ms}$ duration, $0.2-0.4$ $\mathrm{Hz})^{12}$, peripheral mechanical (toothed forceps, Von Frey hairs, brush and air puff) and orthodromic CNS electrical stimuli (MRF; 0.1-4.0 mA, $0.1 \mathrm{~ms}, 0.2-0.4 \mathrm{~Hz}$ ) were used. Mechanical somatic stimuli were applied at intervals of 3-10 s and for durations of approximately $1 \mathrm{~s}$ for each stimulus. Central and peripheral stimuli were delivered with each $100 \mu \mathrm{m}$ movement of the recording electrode. Cocaine- $\mathrm{HCl}$ was administered at an analgesic dose of $25 \mathrm{mg} / \mathrm{kg}$ i.p. in sterile saline. In three experiments, naloxone $(1 \mathrm{mg} / \mathrm{kg}$ i.p.) was administered $15 \mathrm{~min}$ after cocaine injections. The computer program generated multiple trial peristimulus time histograms (PSTH's) and associated statistical parameters. Spontaneous activity was sampled at regular intervals and the evoked response was computed over all stimulus trials as the average number of spikes per second $(\mathrm{sp} / \mathrm{s})$ during the response period. Electrolytic lesions (35-50 $\mu \mathrm{A} D C$ current for $20 \mathrm{~s}$ ) were made at recording and stimulation sites for histological verification.

*Present address: Department of Physiology, University of Helsinki, Helsinki, Finland

Correspondence: K.L. Casey, Neurology Service, VA Medical Center, 2215 Fuller Rd, Ann Arbor, MI 48105, U.S.A. 
Four chronic recording animals were prepared as described previously ${ }^{13}$ with a chronic recording mount stereotaxically positioned above the medial and lateral thalamus. Animals were allowed 2 weeks to recover before recording was initiated. Chloral hydrate (320 $\mathrm{mg} / \mathrm{kg}$, i.p.) was administered just before the recording microelectrode was inserted. A mercury commutator (Dragonfly Industries) was used to minimize cable and movement artifacts. The locomotor effects of cocaine precluded recording from fully awake rats; therefore, all recordings were made while the unrestrained animals were lightly anesthetized and exhibited a robust flexion reflex to hindpaw pinch. Only a single neuron was completely studied in each acutely prepared rat because of uncertainty about the duration of the effects of i.p. administered cocaine. The intensity of noxious stimula-
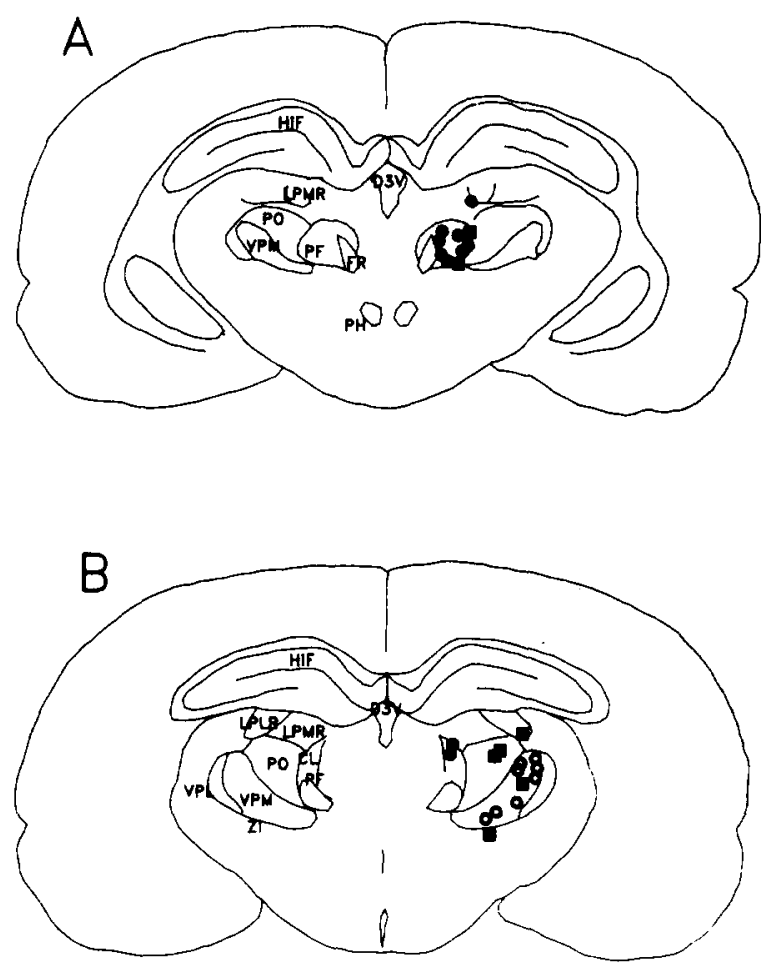

Fig. 1. Anatomical distribution of neurons recorded in the medial and lateral thalamus. Diagrams and abbreviations are modified from Paxinos and Watson ${ }^{15}$. Composite coronal section $A$ is $0.7 \mathrm{~mm}$ caudal to $B$. Twelve of 14 medial thalamic recording sites studied in the acute animal were histologically identified (filled circles). Eleven of these sites are shown on these diagrams; the other was found in the rostral medial portion of the medial dorsal nucleus. Seven neurons were located in the PF, two in CL and two in the LP. Eight lateral thalamic neurons (open circles) were located in the VPM. Of the seven neurons recorded in the chronic preparation (filled squares), two were localized to the PF, two to the $P O$, and one each to VPM, LP and ZI. Cl, central lateral thalamic nucleus; D3V, dorsal aspect of the third ventricle; FR, fasciculus retroflexus; HiF, hippocampal formation; LPLR, lateral posterior thalamic nucleus, laterorostral; LPMR, lateral posterior thalamic nucleus, mediorostral; PF, parafascicular nucleus; PH, posterior hypothalamic area; PO, posterior thalamic nucleus; VPM, ventral posterior medial thalamic nucleus; $Z I$, zona incerta. tion was limited to levels just sufficient to elicit consistent withdrawal movements.

Fig. 1 shows the location of recording sites. In fully anesthetized rats, medial thalamic neurons $(n=14)$ responded exclusively to noxious stimuli (mechanical and electrical). Ninety-two percent (13/14) of the medial thalamic neurons showed reductions ranging from 1 to $160 \mathrm{sp} / \mathrm{s}$ (28-100\%; average, $72.5 \pm 26.1$ S.D.\%) in their nociceptively evoked response within $5 \mathrm{~min}$ of cocaine administration; the remaining cell was unaffected. Control injections of saline were ineffective. Fig. 2 shows examples of the cocaine effect for 3 neurons. Suppression of evoked responses persisted during the $30-90 \mathrm{~min}$ of observation and occurred in the absence of large increases or decreases in the level of spontaneous firing frequency. Mean prestimulus activity before cocaine administration was $3.3 \mathrm{sp} / \mathrm{s}$ (range: $0.22-18 \mathrm{sp} / \mathrm{s}$ ) and the average change was an increase of $0.45 \mathrm{sp} / \mathrm{s}$ (range: $0.15-1 \mathrm{sp} / \mathrm{s})$. Naloxone $(1 \mathrm{mg} / \mathrm{kg}$, i.p.) was administered $15 \mathrm{~min}$ after cocaine without effect in three animals (Fig.

CONTROL

COCANE
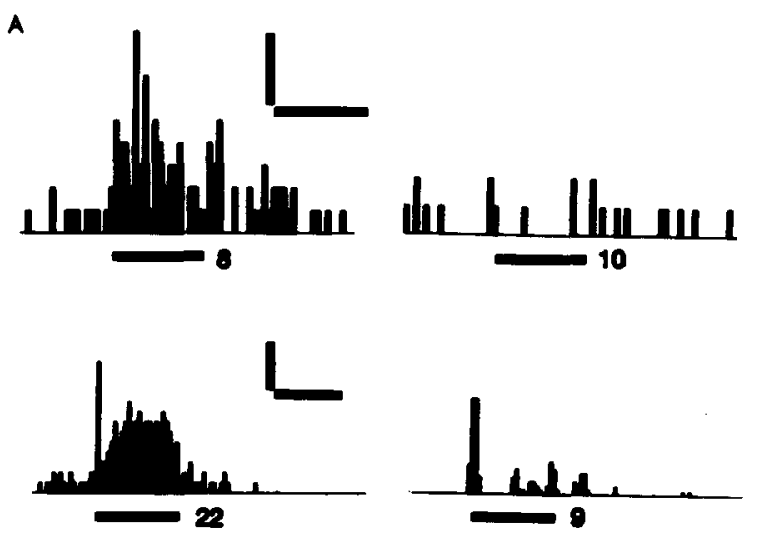

8 CONTROL

COCNNE+
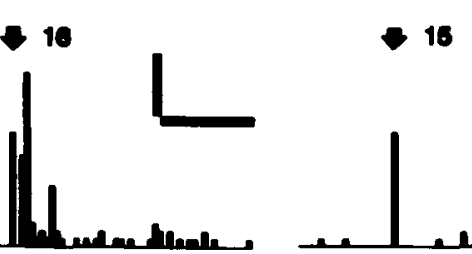

NLOXONE

Fig. 2. A: multiple-trial peristimulus time histograms showing decreased responsiveness of two PF thalamic neurons to noxious forepaw pinch (stimulus bars) $5 \mathrm{~min}$ after cocaine administration to an anaesthetized rat. (Bars: upper, vert. $=0.3 \mathrm{sp} / \mathrm{s}$; hor. $=1 \mathrm{~s}$; bin size $=100 \mathrm{~ms}$; lower, vert. $=0.4 \mathrm{sp} / \mathrm{s}$; hor. $=1 \mathrm{~s}$; bin size $=50 \mathrm{~ms}$ ). Number of trials shown next to stimulus bars. B: parafascicular neuron in the acute, anaesthetized animal showing decreased response to high intensity electrical stimulation $(5 \mathrm{~ms}$ dur., $10 \mathrm{~mA}$, $0.25 \mathrm{~Hz}$ ) of the hindpaw after cocaine administration. Naloxone (1 $\mathrm{mg} / \mathrm{kg}$, i.p.; $15 \mathrm{~min}$ ) did not reverse this effect during the $20 \mathrm{~min}$ of observation after injecting naloxone. Arrow denotes stimulus artifact. (Bars: vert. $=0.5 \mathrm{sp} / \mathrm{s}$; hor. $=0.2 \mathrm{~s}$; bin size $=10 \mathrm{~ms}$ ) Number of trials shown next to stimulus arrows. 
2B). Cocaine also suppressed responses evoked by central electrical stimulation $(0.1 \mathrm{~ms}$ duration, $200 \mu \mathrm{A}$, $0.25 \mathrm{~Hz}$ ) of the MRF in two neurons (CM-PF) that showed a reduced response to peripheral nociceptive stimulation.

All lateral thalamic neurons $(n=8)$ in anesthetized rats responded exclusively to innocuous tactile or pressure stimuli, had small contralateral receptive fields and were all located in the ventroposteriomedial (VPM) region of VB (Fig. 1). Five VPM units showed no change in responsiveness, two showed an increase, and only one showed a significant decrease in the level of evoked activity after cocaine administration. Cocaine had no significant effect on the spontaneous firing frequency of lateral thalamic neurons.

Eight somatically responsive cells were tested with cocaine in the 4 chronically prepared animals. The interval between tests was at least 1 week. Fig. 1 shows that seven histologically localized neurons were recorded from several medial and lateral thalamic nuclei: two each from CM-PF and PO; one each from VPM, LP and ZI. All of the neurons responded exclusively to nociceptive stimuli, and 7 demonstrated an average reduction of 15.5 sp/s (range: $5.9-37 \mathrm{sp} / \mathrm{s} ; 88.4 \pm 7.7$ S.D. \%) in evoked response after cocaine administration. Three neurons showed reductions in noxious-evoked response without changes in their spontaneous firing level; two units showed increases $(8 \mathrm{sp} / \mathrm{s}, 200 \% ; 4 \mathrm{sp} / \mathrm{s}, 100 \%)$ and two showed decreases (1 sp/s, 12\%; $0.35 \mathrm{sp} / \mathrm{s}, 87 \%)$ in spontaneous activity.

The proportion of neuronal responses to noxious stimuli that were reduced by cocaine $(20$ or 22$)$ is

1 Basbaum, A.I. and Fields, H.L., Endogenous pain control mechanisms: review and hypothesis, Ann. Neurol., 4 (1978) 451-462.

2 Belczynski, C.R., Pertovaara, A., Morrow, T.J. and Casey, K.L., Cocaine: neurophysiological effects on bulboreticular projection neurons, Soc. Neurosci. Abstr., 13 (1987) 440.2 p. 1588.

3 Burton, H., Somatic properties of caudal bulbar reticular neurons in the cat (Felis domestica), Brain Research, 11 (1968) $357-372$.

4 Casey, K.L., Somatic stimuli, spinal pathways, and size of cutaneous fibers influencing unit activity in the medial medullary reticular formation, Exp. Neurol., 25 (1969) 35-56.

5 Casey, K.L., Unit analysis of nociceptive mechanisms in the thalamus of the awake squirrel monkey, J. Neurophysiol., 29 (1966) 727-750.

6 Dong, W.K., Ryu, H. and Wagman, I.H., Nociceptive responses of neurons in the medial thalamus and their relationship to spinothalamic pathways, J. Neurophysiol., 41 (1978) 1592-1613.

7 Fields, H.L., Basbaum, A.I., Clanton, C.H. and Anderson, S.D., Nucleus raphe magnus inhibition of spinal cord dorsal horn neurons, Brain Research, 126 (1977) 441-453

8 Fields, H.L. and Basbaum, A.I., Brainstem control of spinal pain transmission neurons, Annu. Rev. Physiol., 40 (1978) 217-248.

9 Guilbaud, G., Peschanski, M., Gautron, M. and Binder, D., significantly different from the proportion of similarly affected responses to innocuous stimuli ( 1 of $8 ; p<0.001$ by Fisher's exact test ${ }^{16}$ ). These results suggest neuronal evoked responses to noxious and innocuous stimuli are differentially influenced by analgesic doses of cocaine. The medial thalamus may be primarily affected by cocaine because the largest proportion of nociceptive neurons was recorded from that site. Neurons recorded in lightly anaesthetized animals also showed decreased responsiveness to noxious stimuli after cocaine. Consistent with behavioral analgesia ${ }^{11}$ and changes in MRF neuronal responses ${ }^{2}$, the effects of cocaine on 3 medial thalamic neurons were not reversible with the opiate antagonist naloxone.

Cocaine produced a suppression or enhancement of thalamic responses without changing the level of spontaneous activity of most neurons, suggesting that thalamic inputs were affected selectively. At least part of the suppression of medial thalamic responses must be attributed to mechanisms acting between the MRF and thalamus because centrally (MRF) evoked thalamic responses were also reduced after cocaine. Because cocaine markedly increases the spontaneous activity of most MRF cells ${ }^{2}$, a monosynaptic effect of MRF cells on the soma of thalamic neurons does not explain the suppressive effect of cocaine on thalamic responsiveness. Additional experiments will be necessary to elucidate the mechanisms underlying these cocaine-induced changes and their significance for cocaine analgesia.

This work was supported by a grant from the Veterans Administration and a Bristol Myers Award, C.R.B. by an NSF Predoctoral Fellowship and A.P. by the Fogarty Foundation.

Responses of neurons of the nucleus raphe magnus to noxious stimuli, Neurosci. Lett., 17 (1980) 149-154.

10 Kevetter, G.A. and Willis, W.D, Collaterals of spinothalamic cells in the rat, J. Comp. Neurol., 215 (1983) 453-464.

11 Lin, Y, Morrow, T.J., Kiritsy-Roy, J.A., Terry, L.C. and Casey, K.L., Cocaine: evidence for supraspinal, dopamine-mediated, non-opiate analgesia, Brain Research, 479 (1989) 306-312.

12 Menetrey, D., Giesler, J. and Besson, J.M., An analysis of the response properties of spinal cord dorsal horn neurons to non-noxious and noxious stimuli in the spinal rat, Exp. Brain Res., 27 (1979) 15-33.

13 Morrow, T.J., Improved technique for recording single unit activity in awake animals, Brain Res. Bull., 5 (1980) 91-93.

14 Morrow, T. J. and Casey, K. L., Suppression of bulboreticular unit responses to noxious stimuli by analgesic mesencephalic stimulation, Somatosens. Res., 1 (1983) 151-168.

15 Paxinos, G. and Watson, C., The Rat Brain in Stereotaxic Coordinates, Academic Press, New York, 1986.

16 Sokal, R.R. and Rohlf, F.J., Biometry, 2nd edn., Freeman, New York, 1981, pp. 740-742.

17 Wolstencroft, J.H., Reticulospinal neurones, J. Physiol. (Lond.), 174 (1964) 91-108.

18 Yezierski, R.P., Gerhart, K.D., Willis, W.D. and Giesler, G.J., Spinothalamic tract neurons that project to medial and/or lateral thalamic nuclei: evidence for a physiologically novel population of spinal cord neurons, J. Neurophysiol., 16 (1981) 1285-1308. 